\title{
Longer-range lattice anisotropy strongly competing with spin-orbit interactions in pyrochlore iridates
}

\author{
L. Hozoi, ${ }^{1}$ H. Gretarsson, ${ }^{2}$ J. P. Clancy,${ }^{2}$ B.-G. Jeon,${ }^{3}$ B. Lee,${ }^{3}$ K. H. Kim,${ }^{3}$ V. Yushankhai,,${ }^{4,5}$ Peter Fulde, ${ }^{4,6}$ D. \\ Casa, ${ }^{7}$ T. Gog, ${ }^{7}$ Jungho Kim, ${ }^{7}$ A. H. Said, ${ }^{7}$ M. H. Upton, ${ }^{7}$ Young-June Kim, ${ }^{2}$ and Jeroen van den Brink ${ }^{1,8}$ \\ ${ }^{1}$ Institute for Theoretical Solid State Physics, IFW Dresden, Helmholtzstr. 20, 01069 Dresden, Germany \\ ${ }^{2}$ Department of Physics, University of Toronto, 60 St. George Street, Toronto, Ontario M5S 1A7, Canada \\ ${ }^{3}$ CeNSCMR, Department of Physics and Astronomy, \\ Seoul National University, Seoul 151-747, Korea \\ ${ }^{4}$ Max-Planck-Institut für Physik komplexer Systeme, Nöthnitzer Str. 38, 01187 Dresden, Germany \\ ${ }^{5}$ Joint Institute for Nuclear Research, Joliot-Curie 6, 141980 Dubna, Russia \\ ${ }^{6}$ POSTECH, San 31 Hyoja-dong, Namgu Pohang, Gyeongbuk 790-784, Korea \\ ${ }^{7}$ Advanced Photon Source, Argonne National Laboratory, Argonne, Illinois 60439, USA \\ ${ }^{8}$ Department of Physics, Technical University Dresden, D-01062 Dresden, Germany
}

(Dated: August 15, 2018)

\begin{abstract}
In the search for topological phases in correlated electron systems, materials with $5 d$ transitionmetal ions, in particular, the iridium-based pyrochlores $A_{2} \operatorname{Ir}_{2} \mathrm{O}_{7}$, provide fertile grounds. Several novel topological states have been predicted but the actual realization of such states is believed to critically depend on the strength of local potentials arising from distortions of the $\mathrm{IrO}_{6}$ cages. We test this hypothesis by measuring with resonant inelastic x-ray scattering the electronic level splittings in the $A=\mathrm{Y}$, Eu systems, which we show to agree very well with $a b$ initio quantum chemistry electronic-structure calculations for the series of materials with $A=\mathrm{Sm}, \mathrm{Eu}, \mathrm{Lu}$, and $\mathrm{Y}$. We find, however, that not distortions of the $\mathrm{IrO}_{6}$ octahedra are the primary source for quenching the spin-orbit interaction, but longer-range lattice anisotropies which inevitably break the local cubic symmetry.
\end{abstract}

PACS numbers:

\section{INTRODUCTION}

It is remarkable that the electronic bands of simple, non-interacting electron systems have intrinsic topological properties which have only recently been uncovered $!^{1 / \sqrt[3]{3}}$ The presence of an insulating state of topological nature has been established in, for instance, a number of bismuth based materials, $\frac{4}{6} 6$ where this state is driven by the strong spin-orbit interaction (SOI) of the rather delocalized bismuth $6 p$ electrons. These materials can be classified as either strong or weak topological insulators (TI's) 7 [ 9

The observed richness of topological states already on the single-electron level prompts the intriguing question what kind of topological phases can develop in more strongly correlated, many-body electron systems. Correlation effects, in particular, intra and inter-orbital electron-electron interactions, are very substantial in $3 d$ transition-metal compounds such as the copper oxides. However, they become progressively weaker when going to heavier transition-metal elements, i.e., $4 d$ and $5 d$ systems, as the $d$ orbitals become more and more extended. Yet the relativistic SOI, the root cause of a number of many topologically non-trivial electronic states, follows the opposite trend - it increases progressively when going from $3 d$ to $5 d$ elements. In $5 d$ transition-metal compounds like the iridates, the interesting situation arises that the SOI and Coulomb interactions meet on the same energy scale. The electronic structure of iridates therefore depends on a strong competition between the elec- tronic hopping amplitudes, local energy-level splittings, electron-electron interaction strengths, and the SOI of the Ir $5 d$ electrons. It is very interesting that the interplay of these ingredients in principle allows the stabilization of entirely novel electronic states such as strong or weak topological Mott states, an axion insulator or a Weyl semi-metallic state $10-14$

In the pyrochlore iridates of the type $A_{2} \mathrm{Ir}_{2} \mathrm{O}_{7}$ that we consider here, with $A=\mathrm{Sm}, \mathrm{Eu}, \mathrm{Lu}$, and Y, five electrons occupy the three $\operatorname{Ir} t_{2 g}$ orbitals, which reside at $\operatorname{Ir}^{4+}$ sites inside corner-linked $\mathrm{IrO}_{6}$ octahedra, see Fig. 1. This leaves one hole in the $t_{2 g}$ shell to which thus 6 distinct $t_{2 g}$ quantum states (3 orbital and 2 spin) are available. When the local symmetry is cubic, so that it does not lift the degeneracy of the three $t_{2 g}$ levels, the strong SOI splits the $t_{2 g}$ states up into a pure $j=3 / 2$ quadruplet and a pure $j=1 / 2$ doublet. The doublet is higher in energy and therefore accommodates the hole. Any additional crystal-field splitting, for instance of tetragonal or trigonal symmetry, lifts the degeneracy of $t_{2 g}$ states and competes with the spin-orbit coupling, thus tending to quench the orbital moment. As the SOI is driving the formation of electronic states of topological nature, the outcome of this competition is decisive for the actual realization of any type of nontrivial topological ground state in pyrochlore iridates. 15116 

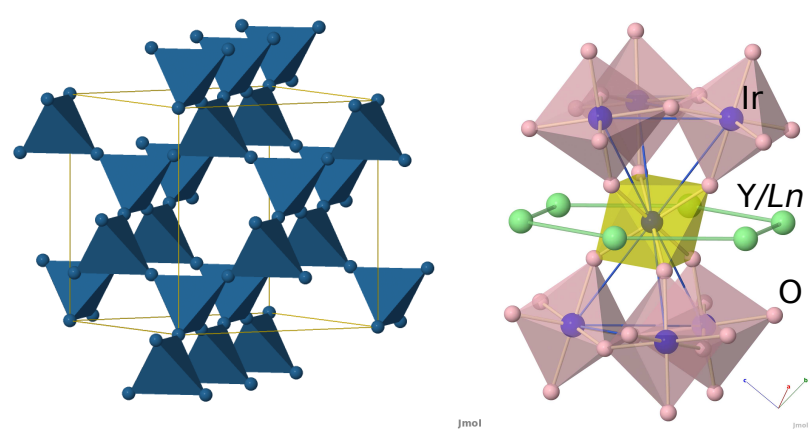

FIG. 1: (a) Network of corner-linked $\mathrm{Ir}_{4}$ tetrahedra in $A_{2} \mathrm{Ir}_{2} \mathrm{O}_{7}$ pyrochlore iridates. (b) A central $\mathrm{IrO}_{6}$ octahedron and how it is connected to its six neighboring $\mathrm{IrO}_{6}$ octahedra. The six trivalent $A$-site atoms form a hexagonal ring around the central octahedron.

\section{EXPERIMENTAL RIXS RESULTS}

We use Resonant Inelastic X-ray Scattering (RIXS) 17 to measure directly the energy of the different configurations of a hole in the Ir $t_{2 g}$ shell 18 of $\mathrm{Y}_{2} \mathrm{Ir}_{2} \mathrm{O}_{7}$ and $\mathrm{Eu}_{2} \mathrm{Ir}_{2} \mathrm{O}_{7}$ and to determine that way the crystal-field energy splittings of these states. The single crystals of $\mathrm{Eu}_{2} \mathrm{Ir}_{2} \mathrm{O}_{7}$ and powder samples of $\mathrm{Y}_{2} \mathrm{Ir}_{2} \mathrm{O}_{7}$ were grown by solid-state synthesis. Mixtures of $\mathrm{Y}_{2} \mathrm{O}_{3}$ and $\mathrm{IrO}_{2}$ with purities of $99.99 \%$ were ground in stoichiometric molar ratios, pelletized, and then heated in air at $1000^{\circ} \mathrm{C}$ for 100 hours. The resulting material was reground, pressed into pellets, and resintered at the same temperature for an additional 150 hours, with two intermediate regrindings. Powder X-ray diffraction measurements confirmed the phase purity of the resulting $\mathrm{Y}_{2} \mathrm{Ir}_{2} \mathrm{O}_{7}$ sample to within the resolution of the measurement. A single crystal of $\mathrm{Eu}_{2} \mathrm{Ir}_{2} \mathrm{O}_{7}$ was grown by the solid-state synthesis method, as previously described in detail in Ref. 19. A mixture of polycrystalline $\mathrm{Eu}_{2} \mathrm{Ir}_{2} \mathrm{O}_{7}$ and $\mathrm{KF}(2 \mathrm{~N})$ were heated up to $1100^{\circ} \mathrm{C}$ and next cooled down to $850^{\circ} \mathrm{C}$ at a rate of $2.5^{\circ} / \mathrm{h}$. Resistivity data on the resulting $\operatorname{Eu}_{2} \mathrm{Ir}_{2} \mathrm{O}_{7}$ single crystal shows almost metallic behavior, indicating that the sample displays slight excess of Ir, see the discussion in Ref. 20 .

RIXS is a second-order scattering technique and can directly probe the electronic transitions within the Ir $5 d$ manifold due to two successive electric dipole transitions $(2 p \rightarrow 5 d$ followed by $5 d \rightarrow 2 p) !{ }^{17118}$ It is therefore a valuable technique for detecting transitions between crystalfield split Ir $5 d$ levels and has been utilized for a variety of iridates $\frac{21}{25}$ We determine the splittings by measuring the $d$ - $d$ transition energies at the iridium $L_{3}$ edge, with an incident energy, $E_{i}=11.217 \mathrm{keV}$, chosen to maximize the resonant enhancement of the spectral features of interest below $1.5 \mathrm{eV}$. It should be noted that varying the incident energy did not result in a shift of any of the peaks but merely changed their intensities, a behavior associated with valence excitations. ${ }^{21}$ 25 The experiments were carried out at the Advanced Photon Source using the 9 ID beamline with a $\mathrm{Si}(444)$ channel-cut secondary monochromator and a horizontal scattering geometry. A spherical (1 m radius) diced $\mathrm{Si}(844)$ analyzer was used and an overall energy resolution of $175 \mathrm{meV}$ (FWHM) was obtained. Higher resolution measurements were carried out using the MERIX spectrometer on beamline 30ID-B. Measurements were performed using a spherical (2 $\mathrm{m}$ radius) diced $\mathrm{Si}(844)$ analyzer and a channel-cut $\mathrm{Si}(844)$ secondary monochromator to give an overall energy resolution (FWHM) of $35 \mathrm{meV}$.

Due to experimental conditions, the spectra for $\mathrm{Y}_{2} \mathrm{Ir}_{2} \mathrm{O}_{7}$ and $\mathrm{Eu}_{2} \mathrm{Ir}_{2} \mathrm{O}_{7}$ were obtained at two different temperatures, 300 and $150 \mathrm{~K}$, respectively. Since the thermal contraction of $\mathrm{Y}_{2} \mathrm{Ir}_{2} \mathrm{O}_{7}$ is extremely small, within tenths of a percentage between 300 and $150 \mathrm{~K}$, and the local oxygen octahedra are unaffected by the temperature, $\stackrel{26}{26}$ we can conclude that this difference in temperature has minimum bearing on our results. The RIXS spectra of both $\mathrm{Y}_{2} \mathrm{Ir}_{2} \mathrm{O}_{7}$ and $\mathrm{Eu}_{2} \mathrm{Ir}_{2} \mathrm{O}_{7}$ in Fig. 2 show sharp features below $1.5 \mathrm{eV}$, corresponding to transitions within the Ir $t_{2 g}$ levels, and a strong intense peak stretching from 2 to $5 \mathrm{eV}$ that according to the calculations, see below, corresponds to $d-d$ transitions between the $\operatorname{Ir} t_{2 g}$ and $e_{g}$ levels. To quantitatively analyze the RIXS spectra, the various peaks were fitted with analytical functions, as shown by the dashed lines in Fig. 2. The low-energy excitations, $E_{1}$ and $E_{2}$, was fitted with one Gaussian and one Lorentzian, respectively. The Lorentzian function was used in order to capture some of the tail on the high energy side $(\sim 1.5 \mathrm{eV})$. The high-energy excitations $\left(E_{3}\right)$ were fitted with a Gaussian peak on top of a sloping background. Such a sloping background may come from charge transfer excitations which are expected to appear in this range.

The position refinement for the three main peaks apart from the zero-loss peak results in $E_{1}=0.53 \pm 0.05$ $(0.59 \pm 0.03), E_{2}=0.98 \pm 0.05(0.97 \pm 0.03)$, and $E_{3}=$ $3.90 \pm 0.05 \mathrm{eV}(3.70 \pm 0.05 \mathrm{eV})$ for $\mathrm{Y}_{2} \mathrm{Ir}_{2} \mathrm{O}_{7}\left(\mathrm{Eu}_{2} \mathrm{Ir}_{2} \mathrm{O}_{7}\right)$. Note that the peak widths are significantly broader than the instrumental resolution, which is about $175 \mathrm{meV}$. To check this, we carried out an additional measurement on the same $\mathrm{Eu}_{2} \mathrm{Ir}_{2} \mathrm{O}_{7}$ sample employing much higher energy resolution of $35 \mathrm{meV}$. This high resolution spectrum is overlayed on top of the low resolution data in Fig. 2. As expected, the improved resolution reveals a little bit sharper features, but still with intrinsic spectral width of about $300 \mathrm{meV}$. We note that the fitted $E_{2}$ peak position of the high resolution data, $0.95 \pm 0.01 \mathrm{eV}$, is slightly smaller than that of the low resolution data, but still within the experimental error bar. Since the high resolution result has smaller error bar, this value is quoted in Table I. We also observed no noticeable momentum dependence for $\mathrm{Eu}_{2} \mathrm{Ir}_{2} \mathrm{O}_{7}$, justifying the use of $\mathrm{Y}_{2} \mathrm{Ir}_{2} \mathrm{O}_{7}$ powder samples for our comparison. 


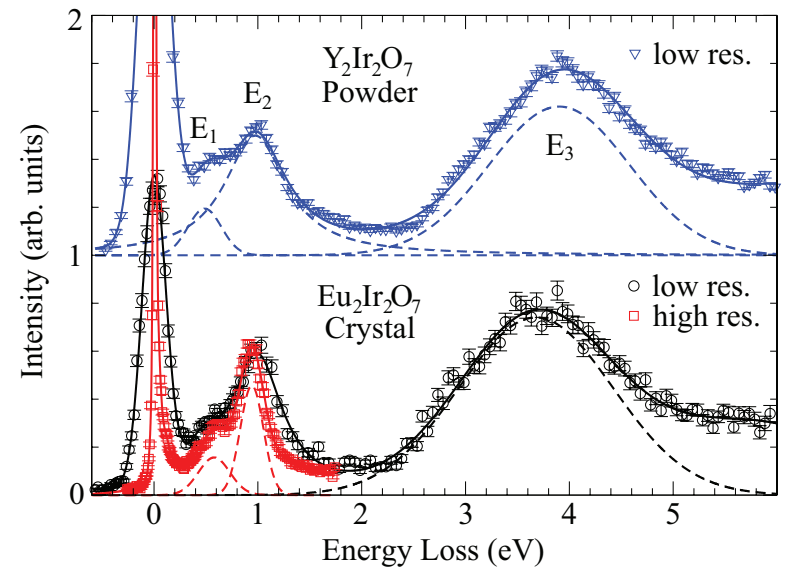

FIG. 2: Resonant inelastic X-ray scattering spectra of $\mathrm{Y}_{2} \mathrm{Ir}_{2} \mathrm{O}_{7}$ (top) and $\mathrm{Eu}_{2} \mathrm{Ir}_{2} \mathrm{O}_{7}$ (bottom) at the iridium $L$-edge. The $\mathrm{Eu}_{2} \mathrm{Ir}_{2} \mathrm{O}_{7}$ spectra were collected at $\mathbf{Q}=(8.45,8.45,7)$. For $\mathrm{Eu}_{2} \mathrm{Ir}_{2} \mathrm{O}_{7}$, high resolution data obtained for low energy region at $(7.8,7.8,7.8)$ are also plotted to emphasize the intrinsic nature of the peak width. Dashed curves are the result of fitting (see text).

\section{EMPIRICAL DESCRIPTION OF RIXS RESULTS}

Using a first, empirical ansatz we fit the energies of the low-lying $E_{1}$ and $E_{2}$ peaks to the eigenvalues of an effective single-ion Hamiltonian for the $t_{2 g}$ orbitals of the form $H_{0}=\lambda \boldsymbol{l} \cdot \boldsymbol{s}-\Delta l_{z}^{2}$, where $\lambda$ is the SOI strength and $\Delta$ the $t_{2 g}$ crystal-field splitting.242728 The latter tends to quench the Ir orbital moment and is usually identified with distortions of the $\mathrm{IrO}_{6}$ octahedra. 15129 The eigenvalues of $H_{0}$ and the splittings among the spin-orbit $t_{2 g}^{5}$ states are given by the following expressions: $E_{0}=\lambda\left(-1+\delta-\sqrt{9+2 \delta+\delta^{2}}\right) / 4, E_{1}=\lambda / 2$, and $E_{2}=\lambda\left(-1+\delta+\sqrt{9+2 \delta+\delta^{2}}\right) / 4$, where $E_{0}$ is the energy of the ground-state spin-orbit $j=1 / 2$ doublet, $E_{1}$ and $E_{2}$ define the eigenvalues of the split $j=3 / 2$-like terms, and $\delta=2 \Delta / \lambda$. If $E_{1}-E_{0}$ and $E_{2}-E_{0}$ are known from experiment, i.e., RIXS, simple estimates for $\lambda$ and $\Delta$ can be in principle derived from the relations above. In particular, $\lambda=2\left(2 E_{10}-E_{20}\right) /(3-\delta)$ and $\Delta=\lambda \delta / 2$, where $\delta=-b-\sqrt{b^{2}-9}, b=\left(1+3 a^{2}\right) /\left(1-a^{2}\right), a=E_{20} /\left(E_{20}-2 E_{10}\right)$, $E_{20}=E_{2}-E_{0}$, and $E_{10}=E_{1}-E_{0}$. The fit of the RIXS data to such a $\lambda-\Delta$ model yields the effective parameters $\lambda=0.43$ and $\Delta=0.56 \mathrm{eV}$ for $\mathrm{Y}-227$ and $\lambda=0.46$ and $\Delta=0.46 \mathrm{eV}$ for Eu-227. The value of $\lambda$ for each of these materials agrees with values of $0.39-0.49 \mathrm{eV}$ extracted from electron spin resonance measurements on $\mathrm{Ir}^{4+}$ impurities. ${ }^{30}$ The magnitude of $\Delta, 0.46-0.56 \mathrm{eV}$, however, is surprisingly large. To understand the size and elucidate the microscopic origin of this large crystal-field splitting - a crucial energy scale in determining the topological ground state of the electronic system - we have carried out a set of detailed $a b$ initio calculations of the Ir $d$-level electronic structure on a series of $5 d^{5}$ pyrochlore
TABLE I: Relative energies (in $\mathrm{eV}$ ) of the split $j=3 / 2$ states $E_{1}$ and $E_{2}$ as well as $t_{2 g}^{5}$ to $t_{2 g}^{4} e_{g}^{1}$ excitation energies in 227 iridates. The results are the outcome of $a b$ initio spin-orbit MRCI calculations, see text. The experimental values for $\mathrm{Eu}_{2} \mathrm{Ir}_{2} \mathrm{O}_{7}$ and $\mathrm{Y}_{2} \mathrm{Ir}_{2} \mathrm{O}_{7}$ determined from their RIXS spectra in Fig. 2 are shown in bold, within brackets.

\begin{tabular}{llll}
\hline \hline & $E_{1}$ & $E_{2}$ & $t_{2 g}^{4} e_{g}^{1}$ \\
\hline & & & \\
$\mathrm{Sm}_{2} \mathrm{Ir}_{2} \mathrm{O}_{7}$ & 0.61 & 0.91 & $3.41-4.75$ \\
$\mathrm{Eu}_{2} \mathrm{Ir}_{2} \mathrm{O}_{7}$ & $0.60(\mathbf{0 . 5 9})$ & $0.91(\mathbf{0 . 9 5})$ & $3.39-4.72(\mathbf{3 . 7 0})$ \\
$\mathrm{Lu}_{2} \mathrm{Ir}_{2} \mathrm{O}_{7}$ & 0.57 & 0.92 & $3.49-4.88$ \\
$\mathrm{Y}_{2} \mathrm{Ir}_{2} \mathrm{O}_{7}$ & $0.58(\mathbf{0 . 5 3})$ & $0.94(\mathbf{0 . 9 8})$ & $3.48-4.84(\mathbf{3 . 9 0})$ \\
\hline \hline
\end{tabular}

iridates: Sm-, Eu-, Lu-, and Y-227.

\section{IV. $A B$ INITIO CALCULATION OF D-D EXCITATIONS}

To investigate in detail the electronic structure and the essential interactions in the $A_{2} \mathrm{Ir}_{2} \mathrm{O}_{7}$ iridates, we rely on $a b$ initio many-body techniques from wavefunction-based quantum chemistry ${ }^{31}$ Multiconfiguration self-consistent-field (MCSCF) and multireference configuration-interaction (MRCI) calculations 31 were carried out to this end on properly embedded finite clusters. Since it is important to accurately describe the charge distribution at sites in the immediate neighborhood,$\sqrt[32]{34}$ we explicitly include in the actual cluster the closest six $A$-ion neighbors and the six adjacent $\mathrm{IrO}_{6}$ octahedra around the reference $\mathrm{IrO}_{6}$ unit for which the Ir $d-d$ excitations are explicitly computed, see also Refs. 24|25|35|37. The solid-state surroundings were further modeled as a large array of point charges fitted to reproduce the crystal Madelung field in the cluster region. All calculations were performed with the MOLPRO quantum chemistry software $\frac{38}{38}$

We used energy-consistent relativistic pseudopotentials for Ir and the $A$ elements $\frac{39}{42}$ and Gaussian-type valence basis functions. Basis sets of quadruple-zeta quality were applied for the valence shells of the central $\mathrm{Ir}^{4+}$ ions ${ }^{39}$ and triple-zeta basis sets for the ligands 43 of the central octahedron and for the nearest-neighbor (NN) Ir sites ${ }^{39}$ For the central Ir ions we also used two polarization $f$ functions ${ }^{39}$ For farther O's around the NN Ir sites we applied minimal atomic-natural-orbital basis sets 44 The $f$ electrons of the $\mathrm{Ln}^{3+}$ species were incorporated in the effective core potentials 40411 and the outer $s p$ shells of the $\mathrm{Ln}^{3+}$ and $\mathrm{Y}^{3+}$ ions were modeled with sets of $[3 s 2 p]$ functions ${ }^{40} 42$ Crystallographic data as reported by Taira et al. ${ }^{45}$ were employed.

For the ground-state calculations, the orbitals within each finite cluster are variationally optimized at the MCSCF level. All Ir $t_{2 g}$ functions are included in the active orbital space ${ }^{31}$, i.e., all possible electron occupations are 
allowed within the $t_{2 g}$ set of orbitals. On-site $t_{2 g}$ and $t_{2 g}$ to $e_{g}$ excitations are afterwards computed just for the central $\mathrm{IrO}_{6}$ octahedron while the occupation of the NN Ir valence shells is held frozen as in the ground-state configuration. The MRCI treatment includes on top of the MCSCF wave functions single and double excitations 31 from the $\mathrm{O} 2 p$ orbitals at the central octahedron and the Ir $5 d$ orbitals.

To extract the local Ir $t_{2 g}$ splittings, the $\mathrm{NN} \mathrm{Ir}^{4+} d^{5}$ ions were explicitly included in a first set of MCSCF calculations. However, the presence of six open-shell Ir NN's makes the spin-orbit calculations cumbersome because for seven $5 d^{5}$ sites (and nondegenerate orbitals), a given electron configuration implies 1 octet, 6 sextet, 14 quartet, and 14 doublet states which further interact via spin-orbit coupling. To simplify the problem and reduce the computational effort, we therefore further replaced the six $\mathrm{Ir}^{4+} d^{5}$ NN's by closed-shell $\mathrm{Pt}^{4+} d^{6}$ ions 57 and in this manner obtained the relative MRCI energies for the spin-orbit states presented in Tables I and II.

The $d$ - $d$ splittings calculated for cuprates such as $\mathrm{La}_{2} \mathrm{CuO}_{4}$ and $\mathrm{Sr}_{2} \mathrm{CuO}_{3} \sqrt[33 / 46 / 47]{ }$ and iridates such as $\mathrm{Sr}_{2} \mathrm{IrO}_{4}, \quad \mathrm{Na}_{2} \mathrm{IrO}_{3}$, and $\mathrm{Sr}_{3} \mathrm{CuIrO}_{6}{ }_{24|25| 35}$ by similar quantum chemistry techniques are in close agreement to the experimental values of these excitation energies. Also for the 227 iridium pyrochlores that we consider here, we observe that the calculated excitation energies of the $5 d$ multiplets (the values of $E_{1}, E_{2}$, and $E_{3}$ ), see Table I, are in close agreement with the ones obtained from our RIXS experiments.

\section{DISCUSSION}

The good agreement between calculated and measured $d$ - $d$ excitation energies forms the basis for a subsequent detailed analysis of the microscopic origin of the crystalfield splitting of the Ir $5 d$ levels. To this end, we first test the hypothesis that the splitting $\Delta$ in the effective single ion $\lambda-\Delta$ model is due to a distortion of the $\mathrm{IrO}_{6}$ octahedra which lowers the local cubic symmetry to trigonal (or even lower) symmetry. It turns out that the crystal structure of the 227's under consideration is fully defined by just three parameters: the space-group number, the cubic lattice constant $a$, and the fractional coordinate $x$ of the $\mathrm{O}$ at the $48 f$ site ${ }^{45}$ For $x=x_{c}=5 / 16$, the oxygen cage around each Ir site forms an undistorted, regular octahedron. In our 227 Ir pyrochlores, however, $x$ is always larger than $x_{c}$, which translates into a compressive trigonal distortion of the $\mathrm{IrO}_{6}$ octahedra and hence a splitting of the $5 d$ electronic levels. To estimate how large the resulting trigonal crystal-field splitting is, we have performed a set of further $a b$ initio calculations, but now for an idealized crystal structure with $x=x_{c}$ and thus undistorted octahedra. The results of the spin-orbit calculations listed in Table II show that the $j=3 / 2$-like states are split off by a sizable amount even for $x=x_{c}$. Thus local trigonal distortions of the $\mathrm{IrO}_{6}$ octahedra are
TABLE II: Calculated energies, $E_{1}^{0}, E_{2}^{0}$, of the $j=3 / 2$-like spin-orbit states in idealized crystal structures without trigonal distortion of the $\mathrm{IrO}_{6}$ octahedra. Those states are split even without trigonal squashing. $\bar{\Delta}^{0}$ and $\bar{\Delta}$ are the splitting of the Ir $t_{2 g}$ levels in MRCI calculations without SOI in the undistorted idealized and experimenta ${ }^{\sqrt{45}}$ crystal structures, respectively.

\begin{tabular}{ccc|cc}
\hline \hline & \multicolumn{2}{c}{ Undistorted Octahedron } & \multicolumn{2}{c}{ Without SOI } \\
\hline & $E_{1}^{0}$ & $E_{2}^{0}$ & $\bar{\Delta}^{0}$ & $\bar{\Delta}$ \\
\hline $\mathrm{Eu}_{2} \mathrm{Ir}_{2} \mathrm{O}_{7}$ & 0.67 & 0.89 & 0.30 & 0.27 \\
$\mathrm{Y}_{2} \mathrm{Ir}_{2} \mathrm{O}_{7}$ & 0.66 & 0.90 & 0.32 & 0.30 \\
\hline \hline
\end{tabular}

not the main cause of the energy splitting $\Delta$ of the Ir $t_{2 g}$ levels.

To understand the physical origin of the large Ir $t_{2 g}$ splittings one needs to consider the crystal structure of the $A-227$ 's in more detail. As shown in Fig. $1 \mathrm{~b}$, the $A$ ions closest to a given Ir site form a hexagonal structure in a plane parallel to two of the facets of the $\mathrm{IrO}_{6}$ octahedron. Even without pushing those two facets of the octahedral cage closer to each other, the six adjacent $A$ cations generate a trigonal field that breaks cubic symmetry. In the simplest picture this positive potential stabilizes the Ir $e_{g}^{\prime}$ orbitals. The latter have the electronic charge closer to the plane defined by the six $A$ NN's, as compared to the $a_{1 g}$ orbital. There is however a competing effect related to the positive NN Ir ions, three above and three below the plane of adjacent $A$ sites, see Fig. 1. The potential generated by the Ir NN's stabilizes the $a_{1 g}$ sublevel. The numerical results we obtain, see below, indicate that the net effect of this anisotropic arrangement of the nearby $A$ and Ir cations on the $t_{2 g}$ level splitting is stronger than the effect of the trigonal distortion of the $\mathrm{IrO}_{6}$ cages. It is interesting to note that similar effects are expected in pyrochlore systems with $3 d$ transition-metal ions. However, the $3 d$ orbitals being more localized will reduce the effect of such anisotropies beyond the first ligand coordination shell and result in smaller splittings of the energy levels.

MRCI calculations without SOI's, see Table II, show that the magnitude of the $t_{2 g}$ splittings is about the same in the distorted, experimental crystal structure $(\bar{\Delta}$, $\left.x>x_{c}\right)$ and the idealized, undistorted structural model $\left(\bar{\Delta}^{0}, x=x_{c}\right)$. This confirms that the splitting of the Ir $t_{2 g}$ levels is due to anisotropic potentials beyond the NN ligand coordination shell. The role of nearby cations in generating anisotropic fields that compete with the trigonal distortion of the ligand cage has been pointed out as early as the 60's for the spinel structure, ${ }^{48 / 49}$ recently confirmed by ab initio quantum chemistry calculations on the $S=3 / 2227$ pyrochlore $\mathrm{Cd}_{2} \mathrm{Os}_{2} \mathrm{O}_{7}$, , 37 and also analyzed for layered Co oxide compounds by densityfunctional calculations.

The calculations on the 227 iridates also show that the $a_{1 g}$ sublevel is lower in energy than the $e_{g}^{\prime}$ sublevels, which is usually referred to as negative trigonal 
splitting. 50151 This indicates that the effect of the positive potential related to the six Ir NN's is stronger than the effect of the field generated by the closest $A$ ions. A stabilization of the $a_{1 g}$ orbital due to positive ions on the trigonal axis has been earlier evidenced by Pillay et al. in $\mathrm{Na}_{x} \mathrm{CoO}_{2}{ }^{50}$ Importantly, if in the quantum chemistry calculations the nuclear charge is artificially lowered by 1 at each of the six Ir NN sites and raised by 1 at each of the six $A$ NN sites, the trigonal splitting changes sign.

While the $a b$ initio calculations without SOI yield $\Delta$ values of $\approx 0.30 \mathrm{eV}$, the fit of the RIXS data with the effective $\lambda-\Delta$ model provides $t_{2 g}$ splittings $\Delta$ of $0.46-$ $0.56 \mathrm{eV}$, more than $50 \%$ larger. This indicates that in pyrochlore iridates the effect of the relativistic spin-orbit coupling cannot be completely captured by oversimplified models such as the $\lambda-\Delta$ Hamiltonian. Additional degrees of freedom must be considered for the construction of a minimal effective model, i.e., hybridization effects, Ir-O and Ir $e_{g}^{\prime}-e_{g}$, in the presence of trigonal external fields and also many-body $d$-shell correlations. The $e_{g}^{\prime}-$ $e_{g}$ couplings, for instance, were found to be important in trigonally distorted $3 d^{5}$ compounds 52 Our data in Table II, showing that with trigonal distortions the $a_{1 g}-e_{g}^{\prime}$ splittings decrease (as $\bar{\Delta}<\bar{\Delta}^{0}$ ), qualitatively confirm the quantum chemistry results of Landron and Lepetit, $[52$ i.e., in the presence of trigonal squashing the $e_{g}^{\prime}$ levels are energetically favored as compared to the $a_{1 g}$ states, in contrast to naive expectations based on one-electron crystal-field theory. Obviously, for $5 d$ oxides, the $t_{2 q}-e_{a}$ couplings are further enhanced by the strong SOI's. 28153

It is also interesting that without trigonal distortions the lower doublet state originating from the $j=3 / 2$ quartet significantly shifts to higher energy as compared to the trigonally compressed experimental structure $\left(E_{1}<\right.$ $E_{1}^{0}$, see Tables II and I), although the splitting of the $t_{2 g}$ levels is about the same in the two cases. The other doublet at somewhat higher energy is on the other hand not much affected. A relevant detail is here that the Ir-O bond lengths are slightly reduced for the data in Table II because the lattice constant was kept the same and in order to remove the trigonal distortion only the fractional coordinate $x$ of the O site was modified. Shorter Ir-O bonds yield higher electron density at the Ir site. The $5 d$-shell Mulliken population,$\sqrt{31}$ for instance, is larger by 0.1 of an electronic charge for the structural model without trigonal distortions. The results of the spin-orbit calculations in Table I and Table II, with a sizable shift of the $E_{1}^{0}$ level to higher energy, indicate that the charge redistribution within the $\mathrm{IrO}_{6}$ octahedron and the higher electron density at the Ir site effectively modify the spinorbit couplings within the $\mathrm{IrO}_{6}$ unit. Feeding the $E_{1}^{0}$ and
$E_{2}^{0}$ quantum chemistry results of Table II to a simple $\lambda-\Delta$ model yields indeed a rather large $\lambda$ effective parameter of $0.49-0.50$ while the corresponding $\Delta$ 's perfectly match this time the $a b$ initio trigonal splittings $\bar{\Delta}^{0}$ computed with no trigonal distortions, 0.30 and $0.32 \mathrm{eV}$. Thus in an idealized system, without trigonal distortions, the effective $\lambda-\Delta$ model provides a reasonable description. It is, however, clear that additional ingredients are required in the effective model for a qualitative description of, e.g., how those excitation energies evolve with the amount of trigonal distortion. This will be the topic of future investigations.

\section{CONCLUSIONS}

We have presented here both experimental and theoretical evidence for the presence of large Ir $t_{2 g}$ splittings in pyrochlore iridates. These splittings arise from longer-range crystal anisotropies that directly compete with spin-orbit interactions. The canonical view that only local distortions of $\mathrm{IrO}_{6}$ octahedra tend to quench the spin-orbit coupling in iridium compounds, in particular iridium pyrochlores, is therefore incomplete. The broader ramification is that the rather extended nature of the $5 d$ wave functions renders the longer-range anisotropy fields to be of fundamental importance throughout the $5 d$ transition-metal series. Their physical effect is particularly striking when the local ligand-field symmetry is high, e.g., cubic or close to cubic, but the point-group symmetry in the crystal is lower. This includes, for example, the osmium-based pyrochlore materials ${ }^{[3754}$ Yet it is pertinent for many more crystal structures, in particular, for layered quasi-2D perovskites or chain-like quasi-1D $5 d$ transition-metal systems. ${ }^{24 \mid 36}$

\section{ACKNOWLEDGEMENTS}

We thank N. A. Bogdanov, V. M. Katukuri, and H. Stoll for fruitful discussions. Research at the University of Toronto was supported by the NSERC, CFI, and OMRI. Use of the Advanced Photon Source at Argonne National Laboratory was supported by the U.S. Department of Energy under Contract No. DEAC02-06CH11357. Work at SNU was supported by the National CRI (2010-0018300) program. L. H. acknowledges financial support from the German Research Foundation (Deutsche Forschungsgemeinschaft, DFG).
${ }^{1}$ C. Kane and E. Mele, Phys. Rev. Lett. 95, 146802 (2005).

2 B. A. Bernevig, T. L. Hughes, and S.-C. Zhang, Science 314, 1757 (2006).

3 M. König, S. Wiedmann, C. Brüne, A. Roth, H. Buhmann,
L. W. Molenkamp, X.-L. Qi, and S.-C. Zhang, Science 318, 766 (2007).

${ }^{4}$ D. Hsieh, D. Qian, L. Wray, Y. Xia, Y. S. Hor, R. J. Cava, and M. Z. Hasan, Nature 452, 970 (2008). 
${ }^{5}$ H. Zhang, C.-X. Liu, X.-L. Qi, X. Dai, Z. Fang, and S.-C. Zhang, Nature Phys. 5, 438 (2009).

${ }^{6}$ B. Rasche, A. Isaeva, M. Ruck, S. Borisenko, V. Zabolotnyy, B. Buechner, K. Koepernik, C. Ortix, M. Richter, and J. van den Brink, Nature Materials 12, 422 (2013).

7 J. E. Moore and L. Balents, Phys. Rev. B 75, 121306 (2007).

8 L. Fu, C. L. Kane, and E. J. Mele, Phys. Rev. Lett. 98, 106803 (2007).

${ }^{9}$ R. Roy, Phys. Rev. B 79, 195322 (2009).

10 H.-M. Guo and M. Franz, Phys. Rev. Lett. 103, 206805 (2009).

11 D. Pesin and L. Balents, Nature Phys. 6, 376 (2010).

12 X. Wan, A. M. Turner, A. Vishwanath, and S. Y. Savrasov, Phys. Rev. B 83, 205101 (2011).

13 L. Balents, Physics 4, 36 (2011).

14 W. Witczak-Krempa and Y.-B. Kim, Phys. Rev. B 85, 045124 (2012).

15 B.-J. Yang and Y. B. Kim, Phys. Rev. B 82, 085111 (2010).

16 M. Kargarian, J. Wen, and G. A. Fiete, Phys. Rev. B 83, 165112 (2011).

17 L. J. P. Ament, M. van Veenendaal, T. P. Devereaux, J. P. Hill, and J. van den Brink, Rev. Mod. Phys. 83, 705 (2011).

18 L. J. P. Ament, G. Khaliullin, and J. van den Brink, Phys. Rev. B 84, 020403 (2011).

19 J. N. Millican, Mater. Res. Bull. 42, 928 (2007).

20 J. J. Ishikawa, E. C. T. O'Farrell, and S. Nakatsuji, Phys. Rev. B 85, 245109 (2012).

21 J. Kim, D. Casa, M. H. Upton, T. Gog, Y.-J. Kim, J. F. Mitchell, M. van Veenendaal, M. Daghofer, J. van den Brink, G. Khaliullin, et al., Phys. Rev. Lett. 108, 177003 (2012).

${ }^{22}$ H. Gretarsson, J. Kim, D. Casa, T. Gog, K. R. Choi, S. W. Cheong, and Y.-J. Kim, Phys. Rev. B 84, 125135 (2011).

${ }^{23}$ K. Ishii, I. Jarrige, M. Yoshida, K. Ikeuchi, J. Mizuki, K. Ohashi, T. Takayama, J. Matsuno, and H. Takagi, Phys. Rev. B 83, 115121 (2011).

24 X. Liu, V. M. Katukuri, L. Hozoi, W.-G. Yin, M. P. M. Dean, M. H. Upton, J. Kim, D. Casa, A. Said, T. Gog, et al., Phys. Rev. Lett. 109, 157401 (2012).

${ }^{25}$ H. Gretarsson, J. P. Clancy, X. Liu, J. P. Hill, E. Bozin, Y. Singh, S. Manni, P. Gegenwart, J. Kim, A. H. Said, et al., Phys. Rev. Lett. 110, 076402 (2013).

26 S. Zhao, J. M. Mackie, D. E. MacLaughlin, O. O. Bernal, J. J. Ishikawa, Y. Ohta, and S. Nakatsuji, Phys. Rev. B 83, 180402 (2011).

27 A. Abragam and B. Bleaney, Electron Paramagnetic Resonance of Transition Ions (Clarendon Press, Oxford, 1970).

28 J. H. M. Thornley, J. Phys. C (Proc. Phys. Soc.) 1, 1024 (1968).

29 G. Jackeli and G. Khaliullin, Phys. Rev. Lett. 102, 017205 (2009).

30 B. Andlauer, J. Schneider, and W. Tolksdorf, Phys. Stat. Sol. B 73, 533 (1976).

31 T. Helgaker, P. Jørgensen, and J. Olsen, Molecular Electronic-Structure Theory (Wiley, Chichester, 2000).
32 C. de Graaf, C. Sousa, and R. Broer, J. Mol. Struct. (Theochem) 458, 53 (1999).

33 L. Hozoi, L. Siurakshina, P. Fulde, and J. van den Brink, Sci. Rep. 1, 65 (2011).

34 L. Hozoi and M. S. Laad, Phys. Rev. Lett. 99, 256404 (2007).

35 V. M. Katukuri, H. Stoll, J. van den Brink, and L. Hozoi, Phys. Rev. B 85, 220402 (2012).

36 N. A. Bogdanov, V. M. Katukuri, H. Stoll, J. van den Brink, and L. Hozoi, Phys. Rev. B 85, 235147 (2012).

37 N. A. Bogdanov, R. Maurice, I. Rousochatzakis, J. van den Brink, and L. Hozoi, Phys. Rev. Lett. 110, 127206 (2013).

38 H.-J. Werner, P. J. Knowles, G. Knizia, F. R. Manby, and M. Schütz, MOLPRO 2010, see http://www.molpro.net

39 D. Figgen, K. A. Peterson, M. Dolg, and H. Stoll, J. Chem. Phys. 130, 164108 (2009).

40 M. Dolg, H. Stoll, A. Savin, and H. Preuss, Theor. Chim. Acta 75, 173 (1989).

41 M. Dolg, H. Stoll, A. Savin, and H. Preuss, Theor. Chim. Acta 85, 441 (1993).

42 K. A. Peterson, D. Figgen, M. Dolg, and H. Stoll, J. Chem. Phys. 126, 124101 (2007).

43 T. H. J. Dunning, J. Chem. Phys. 90, 1007 (1989).

${ }^{4}$ K. Pierloot, B. Dumez, P.-O. Widmark, and B. O. Roos, Theor. Chim. Acta 90, 87 (1995).

45 N. Taira, M. Wakeshima, and Y. Hinatsu, J. Phys.: Condens. Matter 13, 5527 (2001).

46 J. Schlappa, K. Wohlfeld, K. J. Zhou, M. Mourigal, M. W. Haverkort, V. N. Strocov, L. Hozoi, C. Monney, S. Nishimoto, S. Singh, et al., Nature 485, 82 (2012).

47 H.-Y. Huang, N. A. Bogdanov, L. Siurakshina, P. Fulde, J. van den Brink, and L. Hozoi, Phys. Rev. B 84, 235125 (2011).

48 J. C. Slonczewski, J. Appl. Phys. 32, 253S (1961).

49 S. B. Berger, J. Appl. Phys. 36, 1048 (1965).

50 D. Pillay, M. D. Johannes, I. I. Mazin, and O. K. Andersen, Phys. Rev. B 78, 012501 (2008).

51 S. Sugano and Y. Tanabe, J. Phys. Soc. Jpn. 13, 880 (1958).

52 S. Landron and M.-B. Lepetit, Phys. Rev. B 77, 125106 (2008).

53 D. Haskel, G. Fabbris, M. Zhernenkov, P. P. Kong, C. Jin, G. Cao, and M. van Veenendaal, Phys. Rev. Lett. 109, 027204 (2012).

${ }^{54}$ H. Shinaoka, T. Miyake, and S. Ishibashi, Phys. Rev. Lett. 108, 247204 (2012).

55 L. Hozoi, A. H. de Vries, A. B. van Oosten, R. Broer, J. Cabrero, and C. de Graaf, Phys. Rev. Lett. 89, 076407 (2002).

56 R. Maurice, P. Verma, J. M. Zadrozny, S. Luo, J. Borycz, J. R. Long, D. G. Truhlar, and L. Gagliardi, Inorg. Chem. 52, 9379 (2013).

57 This is an usual procedure in quantum chemistry studies on transition-metal systems, see, e.g., Refs. 32375556. 